Peirats Chacón, J. \& Cortés Mollá, S. (2016). El proceso de inclusión en un aula de comunicación y lenguaje. Percepciones de la comunidad educativa. Revista Electrónica Interuniversitaria de Formación del Profesorado, 19 (3), 91-102.

\title{
El proceso de inclusión en un aula de comunicación y lenguaje. Percepciones de la comunidad educativa
}

José Peirats Chacón, Silvia Cortés Mollá

Universitat de València

\section{Resumen}

Actualmente la inclusión es una de las mayores prioridades tanto del ámbito educativo como de la sociedad en general. El trabajo que exponemos se enmarca en el seno del debate abierto en torno a las distintas opiniones y creencias existentes sobre el tema. Concretamente, mostramos los resultados obtenidos en un estudio de caso, al evaluar las percepciones del profesorado y familias del alumnado de un aula de Comunicación y Lenguaje valenciana, sobre el proceso de inclusión realizado a lo largo de sus cinco años de funcionamiento. A través de un diseño de investigación mixto utilizamos instrumentos de recogida de datos cualitativos tales como la entrevista y la observación participante así como de la encuesta entre los cuantitativos, aplicados a una muestra conformada por las familias del alumnado del aula y el profesorado del centro. Entre las conclusiones destacamos que, tanto en las familias como en el profesorado, el aula ha tenido una buena acogida y que se trabaja para mejorar la inclusión de su alumnado; aunque se desprende de las entrevistas y el cuestionario que las respuestas no han sido unánimes y que la acogida del aula y alumnado han provocado reacciones diversas.

\section{Palabras clave}

Educación especial; opinión; familia; profesorado. 


\title{
The inclusion process in a classroom communication and language. Perceptions of educational community
}

\begin{abstract}
At the present time, inclusion is one of the highest priorities of both the education sector and society in general. The work that we present is framed within the ongoing debate about the different opinions and beliefs existing on the issue. Specifically, we show the results obtained in a case study, where we assess the perceptions of teachers and families of the students in a Valencian Communication and Language classroom, about the inclusions process carried out through its five years of functioning. Through a mixed research design, we use qualitative tools as interviews and participant observation and quantitative instruments as surveys, applied on a sample consisted of families of students in that classroom and teachers from the school. The conclusions emphasise that among families and teachers the classroom has been well received and there is a working process to improve the inclusions of the students; although the data collected shows as well that the response has not been unanimous and the host of the classroom and the students has provoked different reactions.
\end{abstract}

\section{Key words}

Special education; opinion; family; faculty.

\section{Introducción}

El concepto de educación inclusiva, surgido hacia los años 90, actualmente se ha convertido en un movimiento de repercusión mundial. Especialmente desde 1994, con la Declaración de Salamanca, donde se proclamó la inclusión como el mejor camino para educar a todo el alumnado dentro de un mismo sistema educativo, teniendo en cuenta todas sus diferencias (UNESCO, 1994). La inclusión educativa, según Echeita y Sandoval (2002), consiste en identificar y minimizar los elementos o barreras que dificultan el aprendizaje y la participación del alumnado, así como tratar de maximizar los recursos que apoyan ambos procesos.

Este discurso no forma parte todavía de la realidad de nuestras escuelas, para lograrlo hacen falta cambios organizativos, didácticos y formativos (Alemany y Villuendas, 2004). Tarea que deben asumir los responsables de la educación, llevando a cabo medidas que garanticen estos principios. Pero no sólo eso, no olvidemos que el éxito de la inclusión depende de numerosos factores: la legislación, la experiencia, los recursos, la formación, las creencias y las actitudes que se tengan sobre ésta (Boer, Pijl, y Minnaert, 2011).

Por esta razón, en estas páginas pretendemos fijarnos en los últimos aspectos citados y plasmar las percepciones de profesorado y familias sobre el proceso de inclusión del alumnado escolarizado en un aula de Comunicación y Lenguaje $(\mathrm{CyL})$. Aspectos a los que Llorens (2012) confiere un papel relevante, al señalar que la introducción de políticas educativas inclusiva en las escuelas ha provocado un impacto en los roles y responsabilidades de los agentes educativos que necesita de análisis y evaluación continua. 
Las aulas CyL son unidades de educación especial ubicadas en los centros ordinarios. Aparecen a partir del curso 2004-05 con carácter experimental en la Comunidad Valenciana, para dar una respuesta educativa adecuada a las necesidades específicas del alumnado con Trastorno del Espectro Autista (TEA) o Trastorno Mixto del Lenguaje (TML), tal como se especifica en las Instrucciones del 23 de Julio de 2014 de la Administración educativa valenciana.

En la normativa citada, aparece como uno de los objetivos de las aulas CyL el favorecer la inclusión de su alumnado. En algunas de las investigaciones aparecidas, como la de Bellver, Díez, López y Navarro (2009), se apunta que la experiencia de estas aulas, si cuentan con los recursos necesarios, es totalmente viable y positiva, debido a la intervención individualizada, la adecuación metodológica y los recursos adaptados. Otros autores (Mascarell y Grau, 2014) consideran que el aula CyL constituye la modalidad de escolarización que, a diferencia del resto, facilita una mayor posibilidad de inclusión escolar y social.

La inclusión educativa es una filosofía de trabajo y una actitud ante la vida que necesita de cierto compromiso de los diferentes agentes de la comunidad educativa. Según Tárraga y Tarín (2013), el camino hacia la inclusión supone una transformación del sistema educativo en general, además de un gran esfuerzo por parte de toda la sociedad. En definitiva, supone un giro copernicano en el diseño y el desarrollo del currículo, la organización escolar y los procesos de enseñanza-aprendizaje (Arnaiz, 2003).

Esta forma de entender la educación ha adquirido mucha relevancia, hasta el punto de llegar a crear un instrumento para su evaluación en los centros educativos, el conocido Index for Inclusion (Booth y Ainscow, 2005). Basado en los principios reflejados en la Carta Internacional de los Derechos Humanos se señala que, para analizar la inclusión, se tendrán en cuenta tres dimensiones: la creación de cultura inclusiva, las políticas inclusivas y las prácticas inclusivas; con el objetivo final de crear comunidades educativas colaborativas e inclusivas.

En el caso del alumnado con TEA, algunas investigaciones han puesto de manifiesto que los ambientes más normalizados proporcionan mayores oportunidades de adquirir mejores habilidades sociales y comunicativas, gracias a estar rodeados de modelos de sus iguales para el aprendizaje (Arce, 2012). Porque el alumnado con TEA, necesita también participar de la vida, aprendiendo a relacionarse e implicarse con los demás (Lozano y Alcaraz, 2012). Sin olvidarnos de las repercusiones positivas que conllevará la presencia de este alumnado en el aula de referencia para el resto de compañeros (Bellver et al., 2009).

Llegado a este punto, nos centramos en una de las variables más importantes en el proceso de inclusión: las percepciones que tienen los agentes educativos. Subraya Fernández (2010), que el profesorado tendrá una relevancia importante pero que no conseguirá llegar al objetivo de la inclusión, si se lleva a cabo sin tener en cuenta las voces y la colaboración del resto de principales agentes educativos.

La actitud hacia la inclusión ha sido estudiada entre el profesorado en sus representaciones y creencias hacia el alumnado con Necesidades Educativas Especiales (NEE). Al respecto, Llorens (2012) apunta que se ven reflejadas en las actitudes en el aula; por tanto, estas creencias se convierten en uno de los principales factores para impulsar el proceso de inclusión. Para concluirlo, esta nueva escuela necesita de un profesorado consciente y comprometido para educar en y para la diversidad (Durán y Giné, 2011).

Sin embargo, el profesorado valora la diversidad de formas muy diferentes; al no existir acuerdo al respecto, el concepto adquiere matices negativos o positivos y surge la 
necesidad de mostrar lo que supone de enriquecimiento para la sociedad. No hay que olvidar que existe un cierto sentimiento generalizado de ansiedad entre los docentes hacia la integración, porque no están preparados ni ayudados por la administración, equipo psicopedagógico o familias (Alemany y Villuendas, 2004).

Podemos decir por tanto, que no hay unanimidad en los estudios que tratan sobre la actitud del profesorado hacia la inclusión, ya que depende de muchos factores relacionados con el tipo de discapacidad, sexo, edad, formación, experiencia, etc. Algunos muestran que, en general, hay una actitud positiva -entre un 65-50-40\%-, aunque tener esa actitud no signifique estar de acuerdo con la inclusión de todo el alumnado con NEE en las aulas (Tárraga y Tarín, 2013).

Es cierto que no se puede imponer al profesorado esta nueva forma de entender la educación, por lo que es necesario trabajar con su colaboración y compromiso. Sin olvidarnos de la formación ya que, como sostiene Arnaiz (2003), hace falta dotar al profesorado de la capacidad técnica para propiciar un cambio de actitudes y poder trabajar la inclusión.

La familia es un contexto esencial tanto para los niños y niñas con discapacidad como para los que no la tienen. Es el referente fundamental que acompaña su crecimiento, por lo que es importante crear un ambiente que ayude a mejorar su desarrollo y potenciar una mejor calidad de vida. Aspecto clave en el proceso inclusivo, porque cuanta más implicación tenga la familia y más comunicación establezca con el centro educativo, mejor será su inclusión.

Conocer lo que entienden las familias sobre la inclusión, ayuda al proceso educativo de sus hijos e hijas. Doménech y Moliner (2013) mediante un relato polifónico con madres de alumnado con NEE muestran sus preocupaciones, percepciones, sentimientos, etc. A través de las distintas conversaciones se refleja la lucha diaria a la que se ven abocadas estas familias defendiendo una educación digna y de calidad, es decir, el modelo de educación inclusiva por el que apuestan.

Tener un hijo con TEA o cualquier otra discapacidad constituye todo un reto, si se asume y no se interpreta como un fracaso parental. En definitiva, tener un hijo con TEA constituye un desafío que requiere abordarse por la aceptación, con el objetivo de compartir juntos el camino de la inclusión.

\section{Método}

Este trabajo está basado en un diseño mixto, utilizado cada vez más en los diferentes campos de las Ciencias Sociales. Las investigaciones de complementación metodológica de los enfoques cuantitativo y cualitativo han ido evolucionando hasta conceptualizarse como una metodología mixta. Sobre la misma, Pereira (2011) sostiene que la utilización de estos diseños constituye una excelente alternativa para abordar temáticas de investigación educativa, otorgándonos la posibilidad de poder profundizar y comprender de mejor forma el fenómeno estudiado.

Mediante esa opción metodológica presentamos los resultados alcanzados en un estudio de caso, realizado en un CEIP con alumnado con TEA o TML en un aula CyL y con parte de su jornada escolar en aulas ordinarias. El objetivo general de la indagación ha sido analizar, a través de cuestionarios y entrevistas, las percepciones de los docentes y las familias sobre el proceso de inclusión de este alumnado.

Instrumentos 
Los cuestionarios favorecen que se pueda abordar el objeto de estudio sobre una muestra considerable de profesorado y familias, utilizando las mismas preguntas se puede construir un mapa general de las percepciones que tienen en torno al tema. De Rada (2002) considera que la encuesta es una búsqueda de información en la que el investigador pregunta sobre los datos que desea obtener, y los reúne para poder obtener diferencias que se localizan en las diferentes personas encuestadas.

En este trabajo los cuestionarios utilizados están construidos mediante preguntas de tipo Likert; la redacción de las preguntas está basada en la observación y en los estudios citados a lo largo del artículo. Para el proceso de validación, se ha seguido el juicio de expertos, que Escobar-Pérez y Cuervo-Martínez (2008), señalan como la opinión informada de personas con trayectoria en el tema que pueden hacer aportaciones importantes: dando informaciones, evidencias, juicios y valoraciones. Para este trabajo, se ha seguido el juicio de seis expertos del ámbito de la educación especial.

Se han realizado dos cuestionarios, uno para el profesorado y otro para los padres y madres del alumnado del aula CyL. Algunas preguntas coinciden, aunque en el caso de las familias el número es más reducido. Para realizar el análisis de fiabilidad se ha utilizado el modelo clásico, el Alfa de Cronbach. Este es un modelo de consistencia interna, indicando el grado de homogeneidad o de consistencia en las respuestas. Está basado en un promedio de las correlaciones entre los ítems, lo que nos informa de la consistencia del conjunto de la escala.

Tabla 1.

Análisis de fiabilidad de las escala

Alfa de Cronbach $\quad \mathrm{N}$ de elementos

Escala percepciones de los maestros

Escala percepciones familias
, 824

17

, 821
11

Como podemos observar existe una fiabilidad alta (tabla n.1) ya que ambas escalas están por encima del valor $\alpha>8$, indicando que el patrón de respuesta por parte de los encuestados es bastante consciente, hecho que avala los resultados obtenidos en el estudio.

En segundo lugar, hemos utilizado también la entrevista semiestructurada, ya que se fundamenta en la producción de un discurso que será continuo, dotándose de una cierta línea argumental, aunque al mismo tiempo fragmentada por la interacción y las preguntas entre el entrevistador y el entrevistado (Sierra, 1998). Es un buen instrumento que ayuda a complementar y enriquecer aquellas opiniones ya expuestas en los cuestionarios. Así hemos creído conveniente realizarla a tres maestros con perfiles diferentes: la maestra de Pedagogía Terapéutica y tutora del aula CyL, un maestro tutor de un aula ordinaria que cuenta con un alumno de aula CyL y otro maestro del centro no tutor y con alumno del aula CyL integrado en sus clases.

Muestra 
Se ha realizado un muestreo no probabilístico, ya que se ha escogido este centro por la vinculación establecida en unos trabajos previos. La muestra está formada por 40 profesionales del centro, de los cuales rellenaron los cuestionarios 28 docentes. Siendo una fracción muestral de $7 / 10$, es decir, un 70\%. Además de realizar las tres entrevistas personales. En el caso de los maestros el $75 \%$ son mujeres, siendo el $25 \%$ restante hombres, y se observa que casi el $50 \%$ de los encuestados han estado formados en Educación Especial. Solo un $25 \%$ ha sido tutor de algún alumno del aula CyL en los cinco años que lleva en funcionamiento, pero casi la mitad de la muestra le ha dado clase $(46,43 \%)$. Además, podemos decir que nos encontramos delante de una plantilla con experiencia, ya que casi un $68 \%$ de los encuestados tienen más de 10 años de docencia y casi un $29 \%$ entre 5 y 10 años. Son muy pocos los que llevan menos de cinco años ejerciendo (3.75\%) y la mayoría de docentes no pasan de cinco años trabajando en el centro.

En las familias, las encuestas se han cumplimentado por núcleo familiar. De los ocho alumnos con los que cuenta el aula, uno es de última incorporación y no se ha utilizado para el estudio. De las siete familias resultantes han contestado a los cuestionarios cinco de ellas. En el caso de familias de padres separados se han facilitado dos cuestionarios, alcanzando finalmente 6 contestadas de las 9 entregadas.

Por último, añadir que se ha realizado la prueba de Kolmogórov-Smirnov con un nivel de significación $p>.05$, siendo que en ambas escalas ha habido una distribución de la población normal.

\section{Resultados}

\section{De las percepciones del profesorado}

Para poder hacer un mejor análisis se han agrupado los ítems según la variable que miden en concreto, de forma que surgen 4 grupos:

a) Percepciones sobre la administración, recursos y la implicación del equipo directivo.

b) Percepciones sobre el funcionamiento del aula.

c) Percepciones del profesorado sobre su tarea con el alumnado del aula CyL, formación y coordinación.

d) Percepciones de cómo afecta a las relaciones entre el alumnado del aula CyL y el resto del alumnado. 


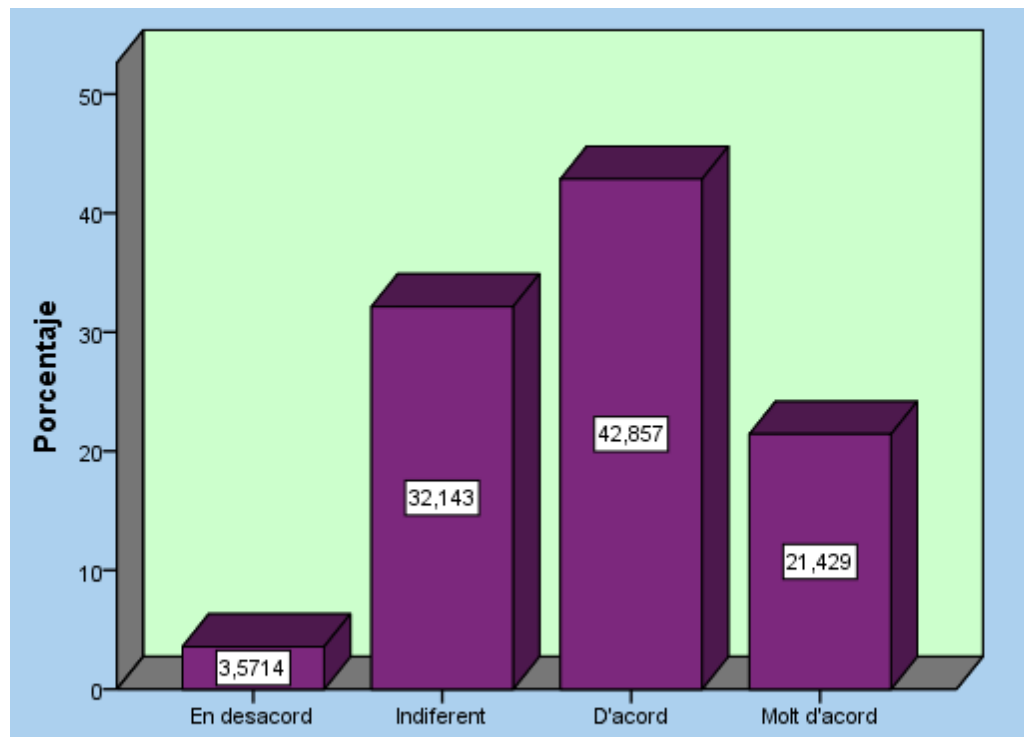

Figura 1. Total percepciones sobre la administración

Pese a haber resultados positivos en la primera variable, encontramos algunos ítems con peor puntuación. Así, aunque hay buenas percepciones en relación a la tarea del equipo directivo, no pasa lo mismo respecto a la administración donde un 26\% y un 19\% están en desacuerdo y muy en desacuerdo en cuanto al fomento de la inclusión por parte de esta institución. Otro ejemplo es que, en relación a los recursos, en las entrevistas la maestra de PT hace referencia al origen de una falta de recursos personales: “¿de quién depende? De la administración". Por su parte, la tutora añade el problema de la identificación y del diagnóstico de los TEA: "no se pueden utilizar los recursos bien, si la selección del alumnado que está en el aula CyL no es la que debería ser".

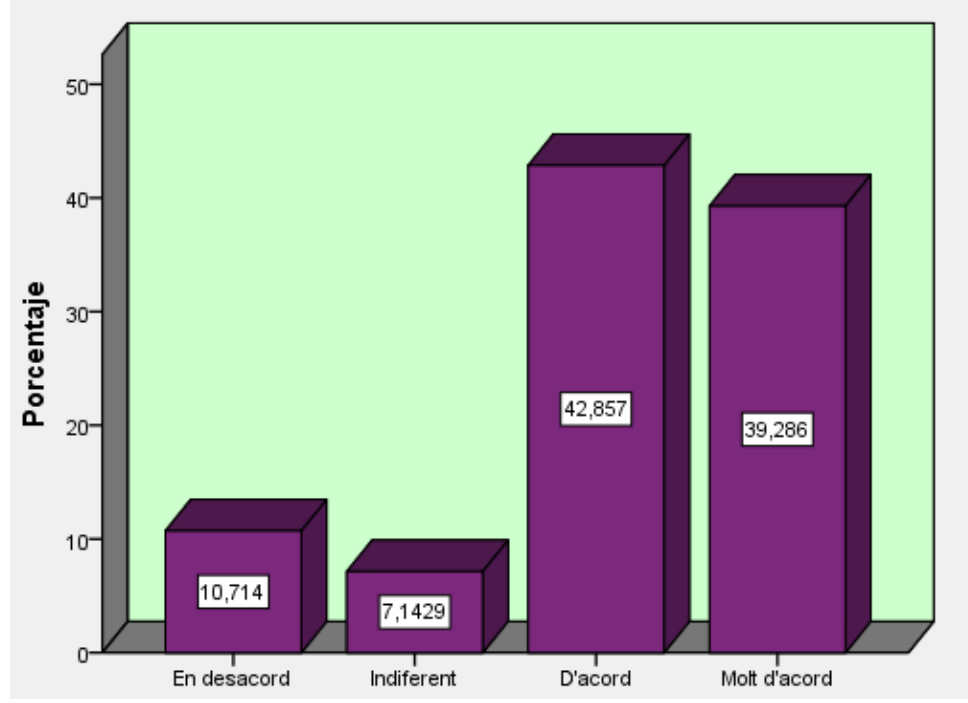

Figura 2. Total percepciones sobre el funcionamiento del aula CyL

Si analizamos la segunda variable, sobre el funcionamiento del aula, aquellas preguntas que hacen referencia directa a la inclusión del alumnado las percepciones son muy buenas, como se puede apreciar en el ítem 8, un 36\% y un 39\% están muy en desacuerdo y en desacuerdo respectivamente en que el aula CyL no tiene tantos beneficios. En la entrevista, la maestra PT señala que el aula CyL funciona y que tiene sentido porque "es mejor que 
estén en un colegio ordinario que no en un específico, aunque no tengan todos los recursos que disponen alli'”.

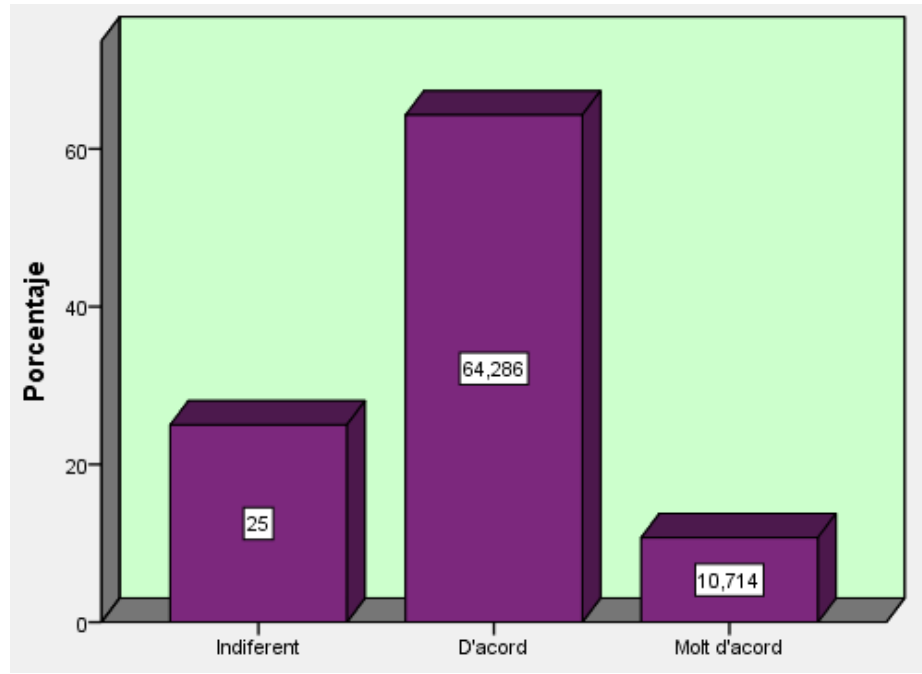

Figura 3. Total percepciones sobre su trabajo en relación al aula CyL

En relación a la variable siguiente, el profesorado sostiene que están de acuerdo y muy de acuerdo en que no tienen la formación necesaria para atender las NEE del alumnado en un $50 \%$ y un $18 \%$, respectivamente. Se evidencia la buena percepción de su trabajo y actitud positiva frente a la inclusión ( $50 \%$ y 32\% de acuerdo y muy de acuerdo respectivamente) pero señalan falta de formación, aunque el $50 \%$ de los encuestados afirma haber recibido formación específica; la tutora afirma: "echo en falta que se nos prepare como tutores que vamos a tener niños con esas características". En cuanto a la coordinación, el ítem 17, se considera que es un aspecto muy importante a tener en cuenta, pero según la maestra PT con el paso del tiempo se ha ido dejando de lado.

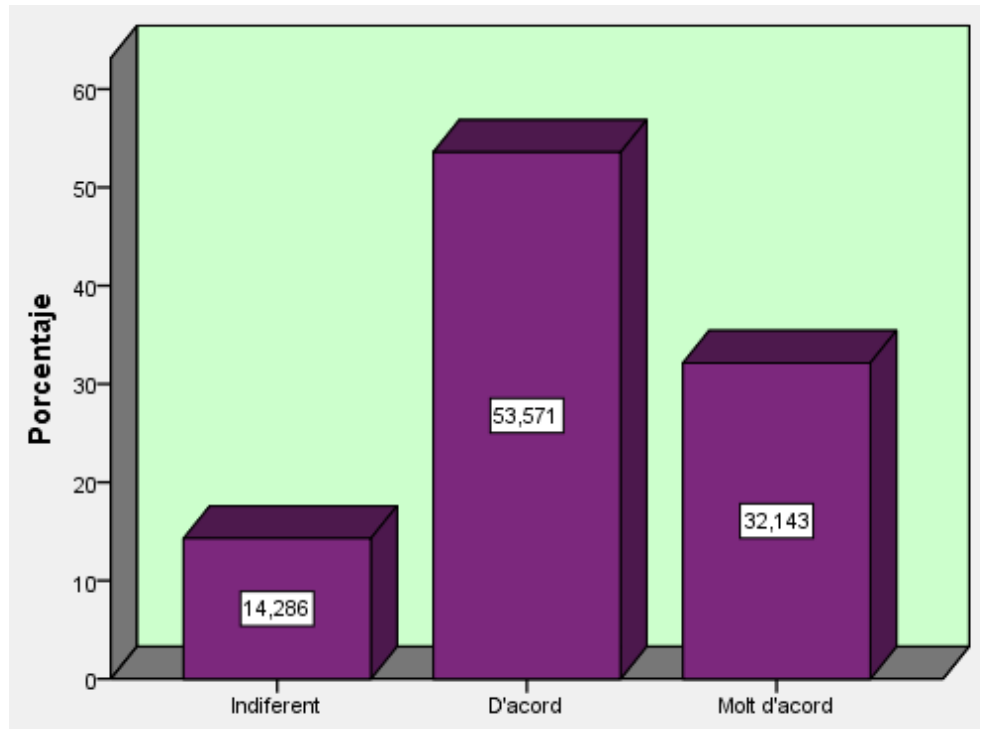

Figura 4. Total percepciones beneficios para el alumnado 
En la última variable, no hay ninguna duda en la percepción positiva hacia que las interacciones potencian la inclusión del alumnado. Así se pone de manifiesto en el ítem 13, donde se afirma que el alumnado del aula $C y L$ implica más beneficios que no inconvenientes, y en las entrevistas se afirma: "no es un trato de protección, sino más bien de respeto o estima". Además, se destaca la importancia de que el alumnado se incluya desde infantil, teniendo una mayor aceptación entre sus iguales.

En definitiva, los resultados sobre la percepción global en cuanto a la inclusión del alumnado del aula CyL en el centro, son muy positivos:

Tabla 2

Percepciones totales profesorado

Frecuencia Porcentaje

\begin{tabular}{lcc}
\hline Indiferente & 6 & 21,4 \\
De acuerdo & 14 & 50,0 \\
Muy de acuerdo & 8 & 28,6 \\
Total & 28 & 100,0 \\
\hline
\end{tabular}

\section{De las percepciones de las familias}

Aquí resaltaremos sólo los ítems más significativos. En referencia al apoyo desde la administración señalar que las percepciones de las familias, a diferencia de las los docentes, sí que son positivas. Pero coinciden con el profesorado en que el equipo directivo también apoya el proceso de inclusión.

En cuanto al funcionamiento del aula las percepciones son muy positivas; en el ítem 5 el $50 \%$ está muy de acuerdo en que el aula ha ayudado a su hijo y un $33 \%$ están de acuerdo. En cuanto a la relación de sus hijos con el resto del alumnado, se muestran de acuerdo en que las interacciones son beneficiosas; podemos observar en el ítem número 11 sobre las interacciones entre el alumnado que el 30\% y el 50\% están de acuerdo y muy de acuerdo, respectivamente, en que son beneficiosas. 


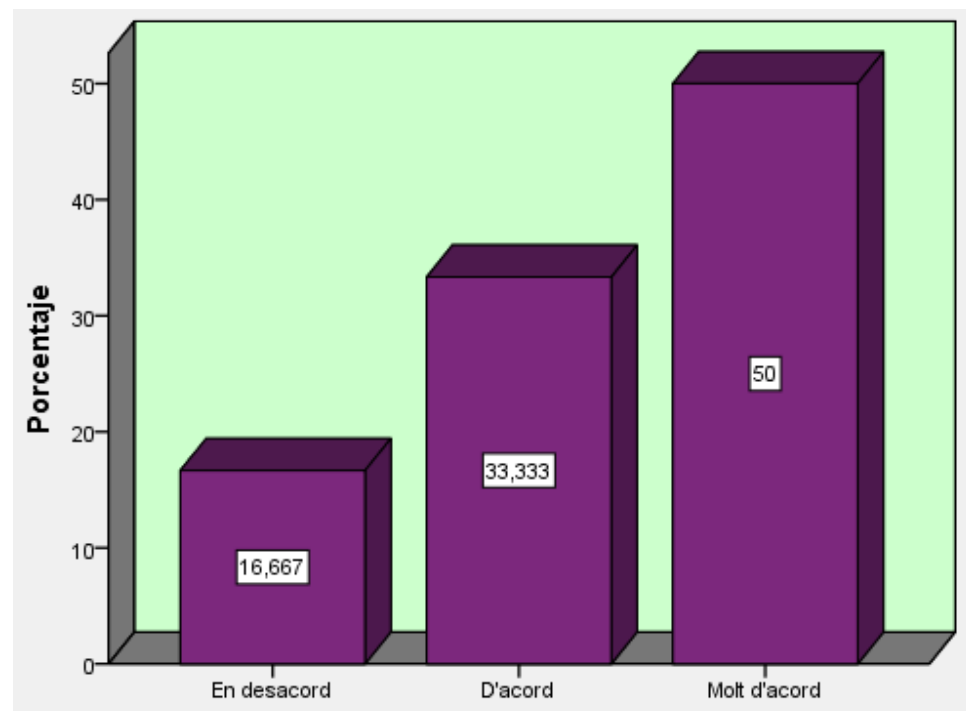

Figura 5. Gráfico de barras ítem 11 interacciones beneficiosas entre alumnado

Por último, las familias están en un $50 \%$ de acuerdo y un $17 \%$ muy de acuerdo en que el centro les da la ayuda necesaria. Pero entre el profesorado entrevistado, en relación a cómo es el trabajo con las familias y si están dispuestas a colaborar, encontramos declaraciones contradictorias, como: "Depende de la familia, si acepta la problemática del nano va a estar más colaboradora, y si no la acepta pues... Es que, si no aceptan en casa lo que tienen, no colaboran. $\mathrm{O}$ no colaboran porque no llegan a más..., o porque llegan y están cansados y con qué coma, duerma y le dé la medicación ya tienen bastante (...)". O la de la tutora al señalar: "La familia está muy implicada y me hace mucho caso, le importa mucho la opinión, mi opinión, me consulta problemas; es decir, se deja un poco llevar por mi opinión y todo lo que le pido lo hace, colabora".

En el caso de las familias, al tener menos preguntas no se han podido agrupar en 4 variables, pero se ha realizado un sumatorio total para ver las percepciones que, en general, también son muy positivas:

Tabla 3.

Percepciones totales familias

Frecuencia Porcentaje

\begin{tabular}{llc}
\hline Indiferente & 1 & 16,7 \\
De acuerdo & 3 & 50,0 \\
Muy de acuerdo & 2 & 33,3 \\
Total & 6 & 100,0
\end{tabular}




\section{Discusión y conclusiones}

Se evidencia, en familias como en profesorado, que el aula ha tenido una buena acogida y que se está trabajando para mejorar la inclusión del alumnado del aula CyL. Pero es cierto que no ha sido una respuesta única y que la acogida del aula y su alumnado han provocado reacciones diferentes. Aunque, como señala la maestra de PT, ha habido una clara evolución desde el inicio del aula hasta ahora, en las reacciones y actitudes del profesorado hacia una mayor aceptación y reconocimiento de la tarea del aula CyL.

Se observan diferencias en algunos puntos, las encuestas y entrevistas han señalado la falta de recursos humanos como un problema importante a tener en cuenta, culpando a la administración no apoya el proceso de inclusión, aunque las familias no coinciden y piensan lo contrario. Por otro lado, el proceso de identificación y la pertinencia o no del alumnado en estas aulas es un tema candente en las entrevistas, ya que como señala la tutora, si no se hace un buen uso de los recursos es lógico que los resultados no sean positivos. También se habla de la falta de formación y las dificultades que supone, a lo que se le añaden los resultados de las encuestas en cuanto al gran desconocimiento que existe entre el profesorado sobre el funcionamiento del aula y sus objetivos.

Otros puntos importantes a señalar son la necesidad de coordinación entre profesionales y con las familias. Señalada la importancia por la literatura (Alemany y Villuendas, 2004; Fernández, 2010; Moya, Martínez y Ruiz, 2005), en las entrevistas se muestra que hay una buena implicación por parte de las familias, o que al menos hacen un esfuerzo importante.

Por último, una apreciación destacable sobre la validez de los procesos inclusivos. Tanto en las entrevistas como en las encuestas se coincide en la mejoría que experimentan cuando se escolariza este alumnado en centros ordinarios, ya que el reconocimiento $y$ acompañamiento se hace desde el primer curso y el resto del alumnado los incluye mejor. Hecho que la maestra de PT relaciona con la importancia de que estén ligados al centro, el barrio o el pueblo; lo que supone discrepancias, dependiendo de la ubicación del centro, con los sistemas de acogida de este tipo de alumnado.

\section{Referencias}

Alemany, I. y Villuendas, M.D. (2004). Las actitudes del profesorado hacia el alumnado con Necesidades Educativas Especiales. Convergencia. Revista de Ciencias Sociales, 11(34), 283-215.

Arce, E. (2012). La inclusión escolar de los alumnos con TEA. Análisis de la respuesta educativa. Comunicación del Conocimiento, 1, 293-308.

Arnaiz, P. (2003). Educación inclusiva: una escuela para todos. Málaga: Aljibe.

Bellver, M.C., Díez, M., López, M.J. y Navarro, A. (2009). La programación didáctica en el aula CYL: La experiencia del CEIP "El Parque de la Cañada" (Paterna). Trabajo presentado en el XIX Congreso Nacional de la Federación Española de Asociaciones de Profesores de Audición y Lenguaje.

Boer, A., Pijl, S. J., \& Minnaert, A. (2011). Regular primary schoolteacher' attitudes towards inclusive education: a review of the literature. International Journal of Inclusive Education, 15(3), 331-353. 
Booth, T. y Ainscow, M. (2005). Índex per a la inclusió. Guia per a l'avaluació i millora de l'educació inclusiva. Barcelona: ICE-UB.

De Rada, V.D. (2002). Tipos de encuestas y diseños de investigación. Pamplona: Universidad Pública de Navarra.

Doménech A. \& Moliner M.O. Families beliefs about inclusive education model. 5th World Conference on Educational Sciences-(WCES 2013). Facoltà di Economia Sapienza Roma, Italy, (380): 05-02-2013.

Durán D. y Giné Cl. (2011). La formación del profesorado para la educación inclusiva. Revista Latinoamericana de Educación Inclusiva, 5(2), 153-170.

Echeita, G. y Sandoval, M. (2002). Educación inclusiva o educación sin exclusiones. Revista de Educación, 327, 31-48.

Escobar-Perez, J. y Cuervo-Martínez, A. (2008) Validez de contenido y juicio de expertos: una aproximación a su utilización. Avances en Medición, 6, 27-36.

Fernández, J. M. (2010). Estrategias y prácticas educativas eficaces para la inclusión educativa: Un estudio de caso en Andalucía. Educational Policy Analysis Archives, 18(22), 1-25.

Lozano, J. y Alcaraz, S. (2012). Respuesta educativa a las personas con trastorno del espectro autista. Madrid: La Muralla.

Llorens, A.J. (2012). Actitud ante la inclusión de niños y niñas con necesidades educativas especiales. Consideraciones de padres, madres y profesionales de la educación. Fòrum de Recerca UJI, 17, 209-227.

Mascarell, A. y Grau, C. (2014). Aula de comunicación y lenguaje en los centros de educación infantil y primaria: estudio de un caso. Trabajo presentado al XI Congreso Internacional y XXXI Jornadas de Universidades y Educación Inclusiva.

Moya, A., Martínez, J. y Ruiz, J.M. (2005). Del aula de educación especial al aula de recursos. Una evolución hacia la inclusión. Innovación Educativa, 15, 119-130.

Pereira, Z. (2011). Los diseños de método mixto en la investigación en educación: Una experiencia concreta. Revista Electrónica Educare, 15(1), 15-29.

Sierra, F. (1998). Función y sentido de la entrevista cualitativa en investigación social. En J. Galindo (coord.), Técnicas de investigación en sociedad, cultura y comunicación (pp. 207-276). México: Addison Wesley Longman.

Tárraga, R. y Tarín, J. (2013). Escuela inclusiva: controversias en torno a discursos, políticas y actitudes. En M.J. Chisvert, A. Ros, y V. Horcas (Eds.), A propósito de la inclusión educativa: una mirada ampliada de lo escolar (pp. 27-53). Barcelona: Ediciones Octaedro, S.L.

UNESCO (1994). Declaración de Salamanca y marco de acción para las necesidades educativas especiales. París: UNESCO. 